Case Reports and Series

\title{
An Evaluation of Surgical Functional Reconstruction of the Foot Using Kinetic and Kinematic Systems: A Case Report
}

\author{
Elena Irene Jordán-Palomar, $\mathrm{MD}^{1}$, Etelvina Javierre, $\mathrm{PhD}^{2}$, José Rey-Vasalo, $\mathrm{MD}^{3}$, \\ Víctor Alfaro-Santafé, $\mathrm{MD}^{4}$, María José Gómez-Benito, $\mathrm{PhD}^{5}$ \\ ${ }^{1}$ Surgeon, Hospital Universitario Miguel Servet, University of Zaragoza, Saragossa, Spain \\ ${ }^{2}$ Associate Professor, Centro Universitario de la Defensa, Academia General Militar, Zaragoza, Spain \\ ${ }^{3}$ Surgeon, Servicio de Cirugía Plástica y Reconstructiva, Complejo Hospitalario de Navarra, Pamplona, Spain \\ ${ }^{4}$ Podiatrist, Podoactiva S.L., Calle Padre Huesca, Huesca, Spain \\ ${ }^{5}$ Associate Professor, Department of Mechanical Engineering, Facultad de Ingeniería y Arquitectura, Aragón Institute of Engineering Research (I3A), \\ Universidad de Zaragoza, Zaragoza, Spain
}

\section{A R T I C L E I N F O}

\section{Level of Clinical Evidence: 4}

Keywords:

pedobarography

plantar flap remodeling

postoperative evaluation

ulcer prevention

weightbearing patterns

\begin{abstract}
A B S T R A C T
Most pedobarographic studies of microsurgical foot reconstruction have been retrospective. In the present study, we report the results from a prospective pedobarographic study of a patient after microsurgical reconstruction of her foot with a latissimus dorsi flap and a cutaneous paddle, with a 42-month follow-up period. We describe the foot reconstruction plan and the pedobarographic measurements and analyzed its functional outcome. The goal of the present study was to demonstrate that pedobarography could have a role in the treatment of foot reconstruction from a quantitative perspective. The pedobarographic measurements were recorded after the initial coverage surgery and 2 subsequent foot remodeling procedures. A total of 4 pedobarographic measurements and 2 gait analyses were recorded and compared for both the noninvolved foot and the injured foot. Furthermore, the progress of the reconstructed foot was critically evaluated using this method. Both static and dynamic patterns were compared at subsequent follow-up visits after the foot reconstruction. The values and progression of the foot shape, peak foot pressure $(\mathrm{kPa})$, average foot pressure $(\mathrm{kPa})$, total contact surface $\left(\mathrm{cm}^{2}\right)$, loading time $(\%)$, and step time (ms) were recorded. Initially, the pressure distribution of the reconstructed foot showed higher peak values at nonanatomic locations, revealing a greater ulceration risk. Over time, we found an improvement in the shape and values of these factors in the involved foot. To homogenize the pressure distribution and correct the imbalance between the 2 feet, patient-specific insoles were designed and fabricated. In our patient, pedobarography provided an objective, repeatable, and recordable method for the evaluation of the reconstructed foot. Pedobarography can therefore provide valuable insights into the prevention of pressure ulcers and optimization of rehabilitation.
\end{abstract}

(c) 2016 by the American College of Foot and Ankle Surgeons. All rights reserved.
The plantar foot consists of highly specialized tissues with fibrous septa that anchor the skin to the underlying fascia to avoid vertical and transversal displacements. The correct and stable position for locomotion is achieved by proprioceptive sensitivity and muscular response. These tissues are arranged to support one's weight and provide balance under static and dynamic conditions. The load supported by the plantar foot is concentrated mainly on the heels and metatarsal heads. This load can be modified by velocity, body weight,

\footnotetext{
Financial Disclosure: None reported.

Conflict of Interest: None reported.

Address correspondence to: Elena Irene Jordán-Palomar, MD, Surgeon, Hospital Universitario Miguel Servet, University of Zaragoza, Saragossa 31008, Spain.

E-mail address: aneleum@gmail.com (E.I. Jordán-Palomar). Video online only at http://www.jfas.org
}

age, and osteoarticular or neurologic pathologic features. With injury to the plantar foot, it is necessary to carefully evaluate the coverage, mechanical stress, and patient characteristics and consider realistic surgical options. Good diagnosis, treatment, and follow-up examinations are essential to provide the patient with the best possible medical care. Normally, injuries to the plantar foot will not lead to the death of the patient; however, they involve long hospital stays, numerous sequelae, and high economic costs to the health care system. In the United States, the estimated average costs when this type of injury affects 1 or both feet are $\$ 21,000$ and $\$ 39,000$, respectively (1).

Injuries to the foot can be difficult to repair, depending on the area involved. Heel tissue reconstruction is complex, because no specific flaps have been biologically designed to cover this particular area. Many surgical options have been introduced, including musculocutaneous flaps, fasciocutaneous flaps, free flaps with or without a 
skin paddle, and perforator flaps (2). Although the criteria for the best type of reconstruction remain unclear $(3,4)$, agreement has been reached that when attempting reconstruction, below-the-knee amputation should be avoided (5). Meyer-Marcotty et al (6) concluded in a retrospective study of 23 patients (with a single measurement per patient) that the long-term results will depend on the exposure of the reconstructed area to pressure. They reported that the ulceration ratio increased from $11 \%$ in non-weightbearing area reconstruction cases to $32 \%$ in weightbearing area reconstruction cases. They used pedobarographic records to show statistically significant differences in the peak pressure on the weightbearing zone between the reconstructed feet and noninjured feet. Moreover, the loss of sensitivity in the affected foot tissues caused scratches, high pressure, bad positions, deformities, and neuropathic ulcers. These pathologic features were the main cause of surgical revision.

In daily practice, postoperative follow-up monitoring of foot reconstruction has often been performed by direct observation or static imaging methods, such as radiography or computed tomography. However, since the first reports by Goldberg et al (7), who recorded plantar footprints by soaking the plantar foot with mineral oil, asking the patient to step onto an absorbent type of paper, and drawing the resulting contour on the paper with a marking pen, more objective and evaluator-independent methods have been sought. Villa Moreno et al (8) studied alterations in weightbearing patterns and gait using kinetic, kinematic, and electromyographic analyses to establish benchmarks for future comparisons.

Öztürk et al (9) used similar pedobarographic methods to evaluate the functional outcomes after lower limb reconstruction in patients who had sustained serious injuries after landmine explosions. The investigators retrospectively compared 72 cases of plantar foot reconstruction at a single point with a group of 20 healthy patients (9). Dynamic pressure distribution tests revealed significantly higher pressure and loads on the injured feet. Three-dimensional motion analyses showed a restricted range of motion of the ankle joints in the injured extremities. This is the longest series of cases to document the long-term functional results of patients injured by landmines and treated with free muscle flaps. The investigators concluded that reconstructive options should be preferred over amputation procedures, whenever possible, in cases of extensive tissue loss caused by landmine explosions (9).

The aim of the present study was to report a prospective model to record and evaluate the functional results before and after foot surgery. We describe a single clinical case in great detail. To the best of our knowledge, pedobarography has not been previously used to evaluate functional outcomes after foot surgical reconstruction and to plan rehabilitation. The results were also used to design specific insoles for the patient. These were fabricated using rapid prototyping to compensate for the pressure distribution differences in the plantar foot.

\section{Case Report}

The present work describes the case of a 32 -year-old white female, with no relevant surgical or personal history, who had experienced a motorbike traffic accident in November 2009. The timelines of the different surgical procedures and the acquisition of biomechanical data are presented in Fig. 1 and the Table. When crushed by the side of the motorbike, the patient's right foot had sustained serious injuries; however, her other injuries were minor. They included superficial abrasions and bruises on the arms. At hospital admission, the patient presented with a Lisfranc fracture dislocation, a fifth metatarsal fracture, and subfascial degloving of the plantar foot with a distal pedicle. She was admitted to the orthopedic surgery department and fixation of the fractures and wound dressing changes were performed. The patient had no other serious injuries. After 6 days, the patient had developed a posterior tibial artery thrombosis, which caused cutaneous, fascial, and fat tissue necrosis of the heel and plantar arch area (Fig. 2).

The plastic surgery department was then consulted for debridement and coverage of the involved areas. The patient was informed and provided written consent for her participation in a prospective study. We evaluated the preoperative and postoperative functional results of plantar foot reconstruction through

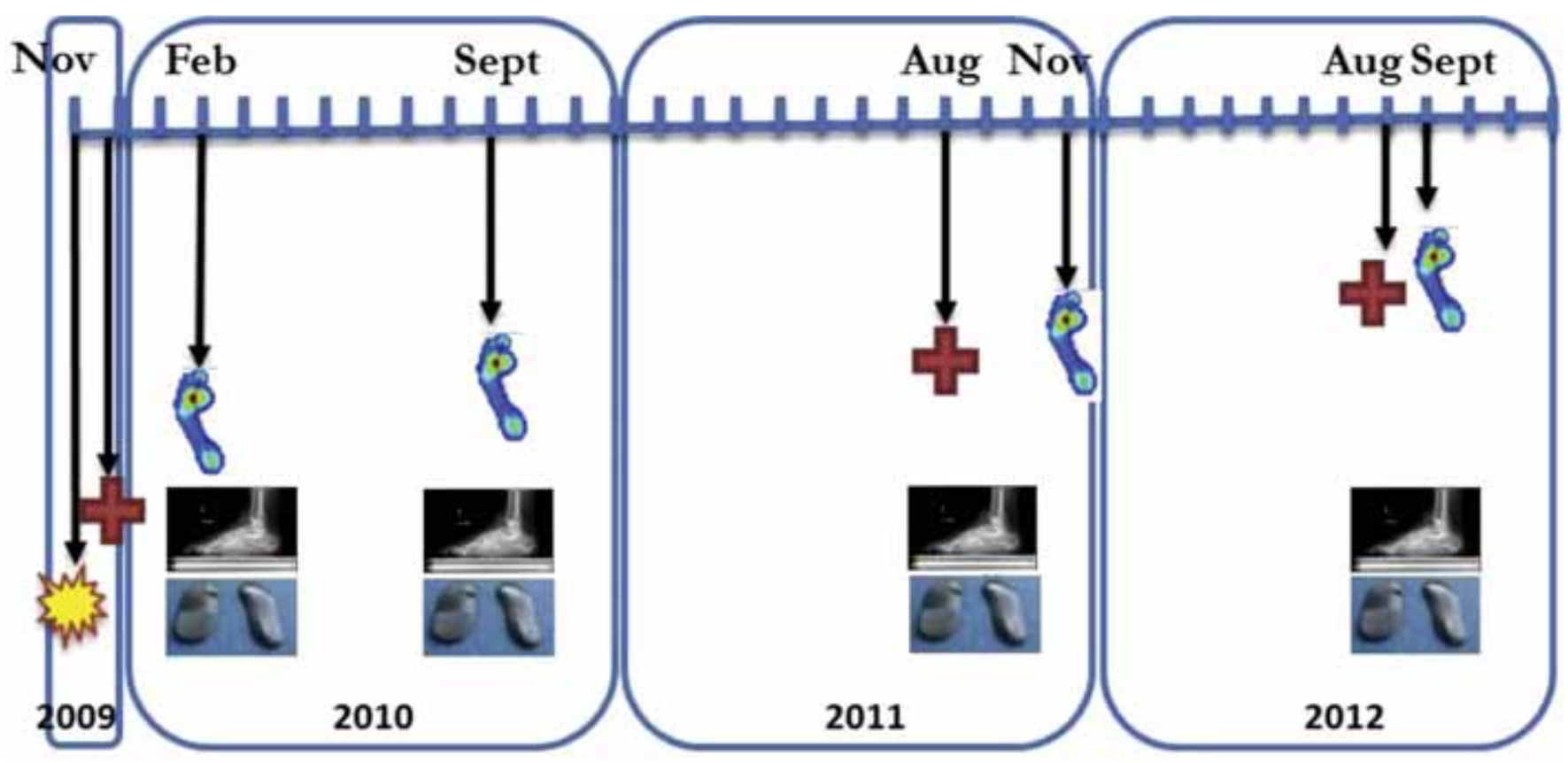

Fig. 1. Timeline of patient follow-up care. Red crosses indicate surgical intervention. Photograph enlarged above. 
Table

Timeline of the different measurements used in the present study

\begin{tabular}{|c|c|c|c|c|}
\hline Variable & $\begin{array}{l}\text { February } \\
2010\end{array}$ & $\begin{array}{l}\text { September } \\
2010\end{array}$ & $\begin{array}{l}\text { November } \\
2011\end{array}$ & $\begin{array}{l}\text { Septembe } \\
2012\end{array}$ \\
\hline \multicolumn{5}{|l|}{ Static and dynamic analyses } \\
\hline Without compression stocking & Yes & Yes & Yes & Yes \\
\hline With compression stocking & No & Yes & No & Yes \\
\hline Gait analysis & No & No & Yes & Yes \\
\hline
\end{tabular}

the measurement of maximal and mean pressures, contact surface, time of contact, spatial distribution of pressure, and footstep trajectory.

The plastic surgery team operated on the patient several times. The first surgery was performed on her arrival at the hospital in November 2009, 20 days after the accident. At that time, debridement of the necrotic tissues and coverage of the heel and plantar arch with a free latissimus dorsi flap with a cutaneous paddle were performed. The flap was fixed to the bones with mini quick anchor Mitek ${ }^{\circledR}$ (Depuy Synthes, West Chester, PA), and partial thickness grafts were placed in the dorsal zone. The bone fractures were fixed with Kirschner wires. The patient had no postoperative complications, and she was discharged home 10 days after surgery. The patient was not allowed to apply loads to the affected foot for 6 weeks, until radiologic signs of bone healing were evident. The patient subsequently began to walk with the aid of crutches.

The clinical course of the patient was faster in the first months after the first surgery and then it stabilized. The first pedobarographic measurements were obtained in February 2010, 3 months after the first surgery, when the patient could apply a full load to her feet. Subsequently, systematic pedobarographic and gait analysis measurements were obtained according to the timelines presented in the Table and Fig. 1. These tests were performed free of charge by an independent company not affiliated with our hospital and located in a different city. The second pedobarographic measurements were obtained in September 2010, when all the tissues were stable and the amount of inflammation was low. This second set of measurements was considered a reference for the "normal" situation after the first surgery. The second surgery was postponed until August 2011 because of personal matters of the patient. However, from a clinical perspective, the surgery could have been performed several months earlier, when we had performed the pedobarographic measurements.

In August 2011, we performed the second surgery. At that time, fat tissue from the plantar area was excised, and liposuction of the dorsolateral area was performed. This surgery was planned according to the results observed in the pedobarographic study, at which an excess volume in the plantar arch area was observed. Again, 3 months later (November 2011), a new pedobarographic study was performed to allow the tissues to stabilize after surgery. The patient had a satisfactory clinical outcome, but she referred to excess volume in the foot dorsum that had made shoe adaptation difficult and produced undesirable aesthetic results. Thus, in August 2012, the cutaneous excess in the dorsum of the foot was removed without performing any changes to the plantar foot, with the aim of not modifying the functional results achieved with the previous surgery. The results were evaluated 1 month after surgery (no surgery was performed on the plantar foot) using a new set of pedobarographic measurements in which we aimed to disregard the occurrence of unwanted changes in the foot sole support. During the complete follow-up period, 3 different personalized insoles were created for the patient using fast prototyping to improve the plantar foot support and prevent ulceration.

Static and dynamic pedobarography tests were always conducted with the patient barefoot. The compression stocking was used in 2 measurements to check its functionality. The patient also wore a compression stocking and insoles in her daily life to compensate for the abnormalities observed during the tests (Fig. 3).

Gait analysis tests were conducted using 2 different methods, with the patient barefoot and with the patient wearing shoes and the specifically designed insoles. Neither crutches nor canes were used for the acquisition of the presented data. The techniques used for the systematic collection of the data are presented in subsequent sections.

\section{Pedobarography}

The plantar foot pressure distributions were recorded under static and dynamic conditions using the FootWork ${ }^{\circledR}$ system (Fig. 4A) at the facilities of Podoactiva S.L. (Walka Technology Park, Huesca, Spain). FootWork $^{\circledR}$ is a tool designed to detect alterations in the pressure distribution. Static position measurements were obtained with the patient standing for 4 seconds (Supplemental Video S1). Dynamic measurements were obtained with the patient walking on the platform (Supplemental Video S2).

Laboratory recordings were performed using video, and the presented results are a summary of all the sequences and represented as a single image. This system is able to locate the areas at a greater risk of wounding. Thus, it can be used to define the best strategy to compensate for the pressure distributions $(11,12)$.

\section{Gait Analysis}

Gait quantitative data were recorded with the OptoGait ${ }^{\circledR}$ modular system (Woodway World, Waukesha, WI; $10 \mathrm{~m}$ with 2 barriers of photocells). With this system, the patient walks freely several times over the gait corridor (Fig. 4B). The system records and compares the patient's gait functional parameters to those from a normal population database. Nevertheless, it does not provide an etiologic diagnosis or the precise anatomic location of the injury. A high-resolution camera was also used to record the sequence to allow for posterior visual analysis of the gait abnormalities $(13,14)$.
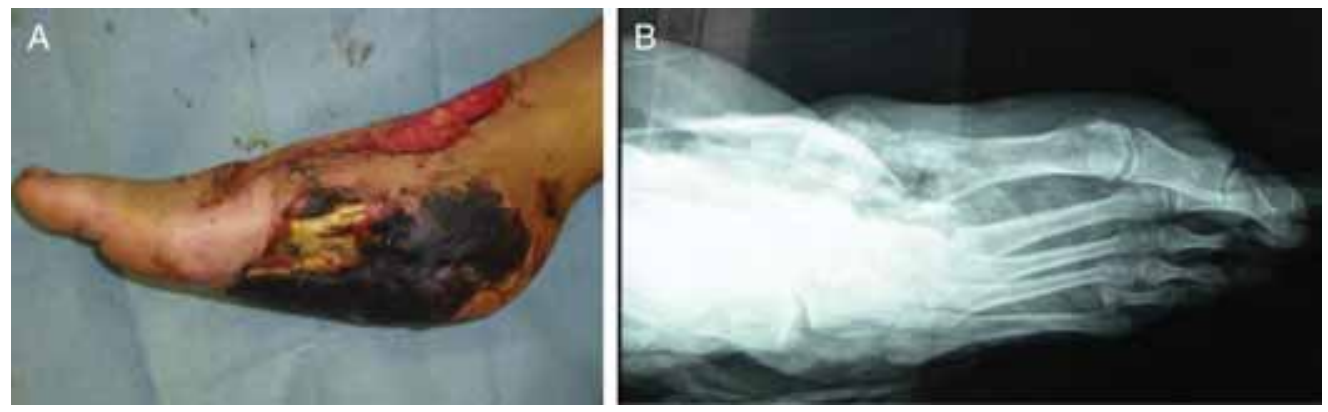

Fig. 2. (A) Preoperative appearance and $(B)$ radiograph of the affected foot 20 days after injury. 


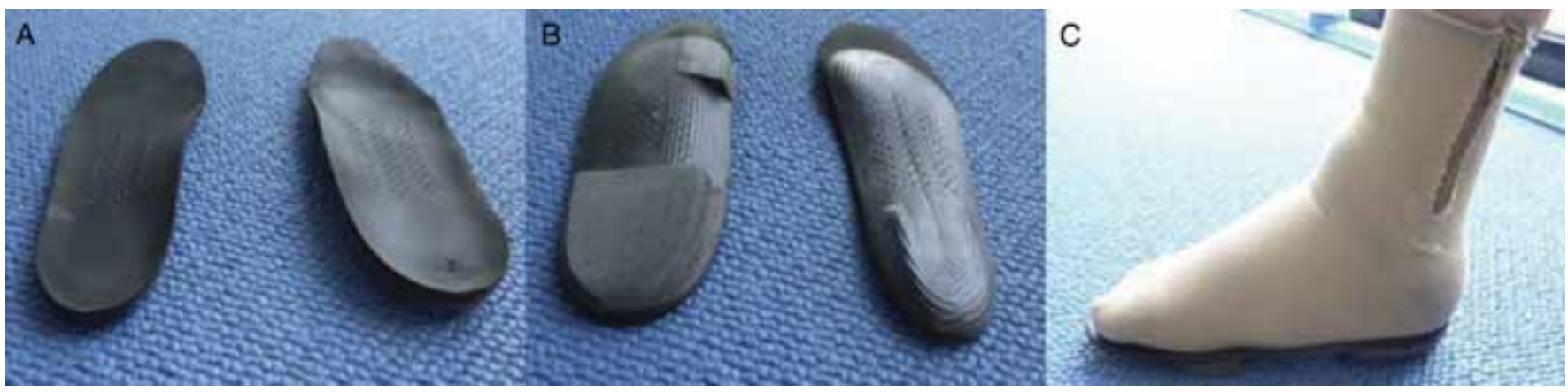

Fig. 3. ( $A$ to $C$ ) Rapid prototyped insoles and compression stocking.

We followed up the patient from the functional and clinical perspectives (Fig. 5). The nonaffected foot presented 2 weightbearing points in a static situation: the heel and the metatarsal area. In a dynamic situation, the weightbearing point changed with the addition of the outer border of the foot and toes to the weightbearing areas. The gait sequence began with weightbearing by the heel. Subsequently, a smooth rotational arc occurred that displaced the weight toward the outer border of the foot. Finally, the weight was supported by the head of the first metatarsal. In our patient, the nonaffected foot's dynamic footprint revealed an increase in the load of the second metatarsal. This indicated that the foot was cavus and had a squared shape, a short first toe, and a tendency to supination. Moreover, this foot tried to compensate for the wrong position of the affected foot, displacing the load to the heel to reach equilibrium.

The center of equilibrium of the nonaffected foot oscillated less than that of the affected foot. In successive recordings, these values were regularized: the distance to the center of equilibrium of the body decreased progressively, tending to be closer to both feet. We analyzed in detail the results from the affected foot over time.

\section{February 2010}

The first laboratory mechanical evaluation of the patient's progress after the coverage surgery was performed in February 2010. At that time, the flap was rather large and inflamed, causing the reconstructed foot to have the appearance of an unstable oscillating hemisphere. When the patient stood still on both feet (static position), the contact surface of the affected foot was very small, and the

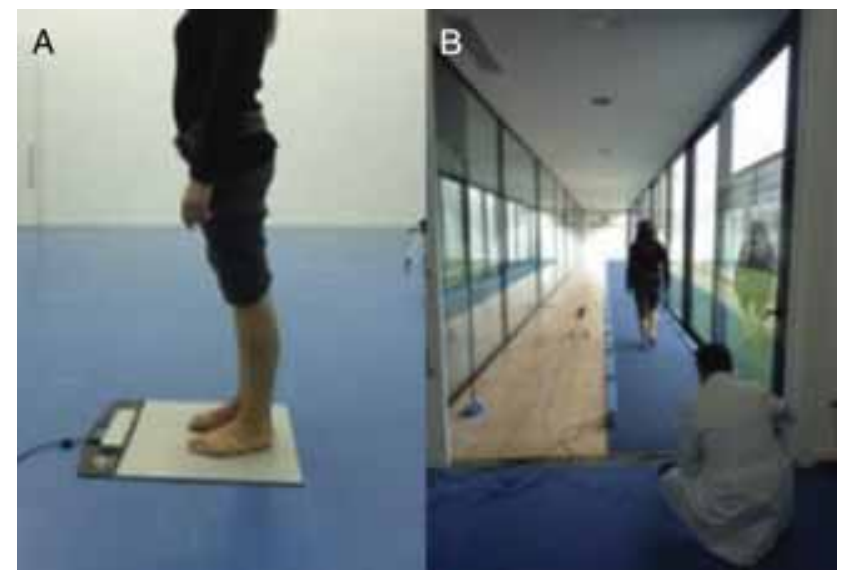

Fig. 4. Measurements of foot pressure distributions using FootWork ${ }^{\circledR}(A)$ and gait parameters using OptoGait ${ }^{\circledR}(B)$ greatest pressure was concentrated in the central area of the plantar foot, over the flap. Furthermore, the toes did not support any load (Fig. 5 [5.1]). Additionally, the patient tried to compensate for the instability of the affected foot by displacing the weight toward the anterior area (which conserved sensibility and good proprioception). The nonaffected foot compensated for this weight shift by moving slightly backward. The results of the affected foot dynamic footprint (Fig. 5 [5.2]) showed a straight step trajectory with great oscillations in the central area, markedly different from the step trajectory of the noninjured foot. This load distribution indicated that certain areas of the affected plantar foot bore elevated pressure for prolonged periods. This could have triggered pain and ulceration. Therefore, rest was recommended to the patient.

\section{September 2010}

In the period from February 2010 to September 2010, the amount of foot inflammation and pain had decreased. Furthermore, the patient's self-learning had helped with this improvement. In the static position, the contact area and balance of the affected foot had increased (Fig. 5 [5.3]). Moreover, the distances from both feet to the center of balance were similar. In the dynamic situation, a change in the footprint shape (from oval to a paddle shape) was observed (Fig. 5 [5.4]). The affected foot's step trajectory had become slightly arched. Moreover, the pressure distribution of both feet was similar, and, for the first time, a small footprint of the toes on the affected foot had appeared. Still, excessive soft tissue contact appeared in the plantar arch area. Therefore, surgery to reduce this nonphysiologic support of the plantar arch was planned.

\section{November 2011}

In August 2011, a surgical intervention was conducted on the affected foot to reduce its plantar foot volume and to remove the excess fat in the plantar arch. Therefore, the results obtained in November 2011 represent the functional postoperative outcome. The results of the affected foot in the static position (Fig. 5 [5.5]) showed a more homogeneous pressure distribution and displacement of the peak pressure from the heel to the medial plantar area. The results of the dynamic footprint (Fig. 5 [5.6]) of the affected foot showed, for the first time, a physiologic arc in the step trajectory. Furthermore, the pressure distribution showed great improvement: all the toe footprints had appeared, the contact of soft tissues in the plantar arch area had decreased, and the peak pressure had appeared in the metatarsal area.

\section{September 2012}

Another surgery was conducted in August 2012 to remove the dorsal skin and fat to reduce the global foot volume and improve the 


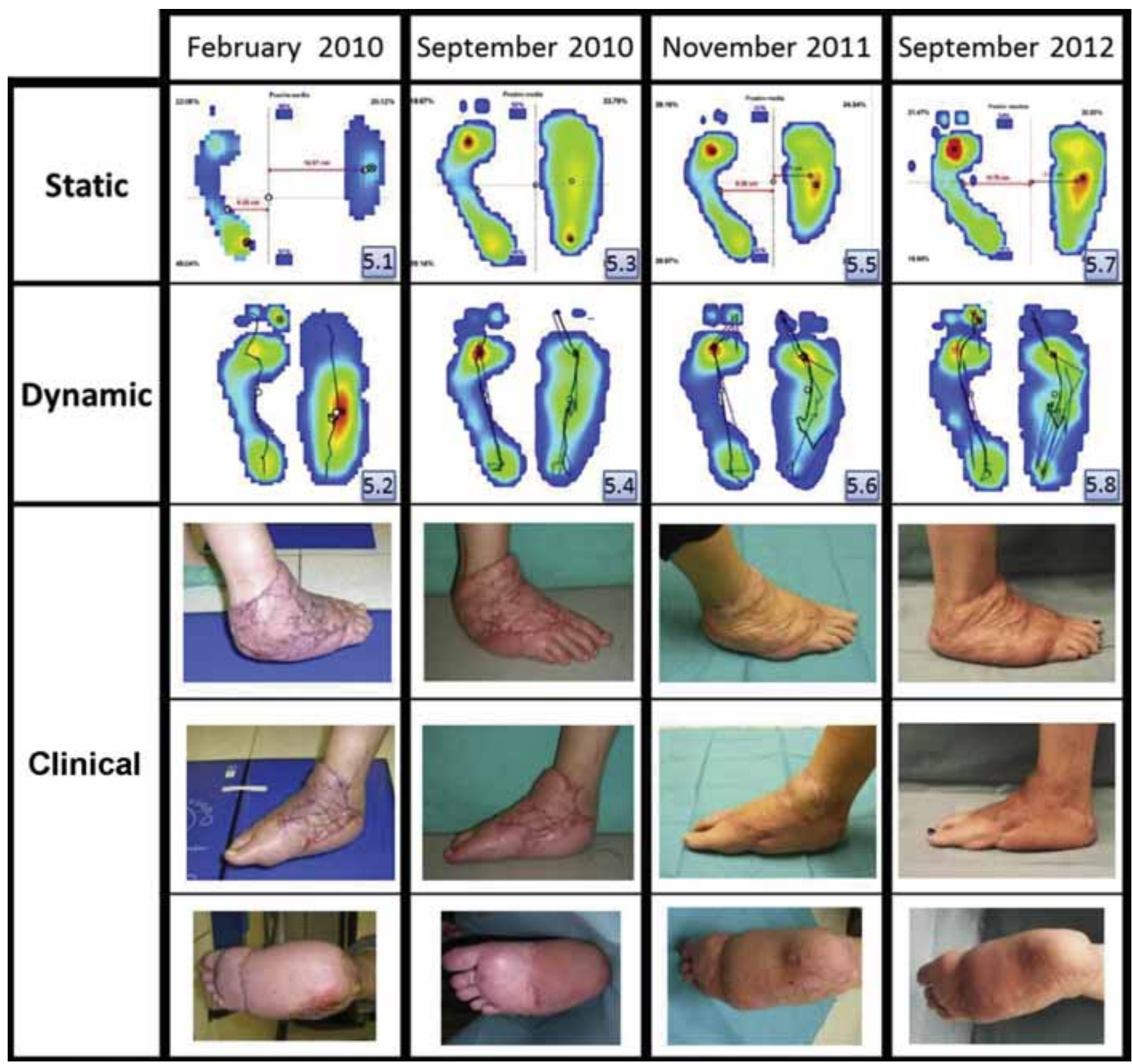

Fig. 5. Temporal evolution of weightbearing distributions under static and dynamic conditions and clinical evolution of the affected foot. Contour plots show lowest pressures in blue and highest pressures in red. Crossed circle indicates the position supporting the highest pressure.

aesthetic aspect. In addition, the skin on the external lateral side of the foot was removed to give more tension to the skin of the plantar foot. This surgery did not directly modify the plantar foot surface. Again, the results discussed in this section represent the functional postoperative outcome. A greater and more homogeneous pressure distribution was obtained in the static analysis (Fig. 5 [5.7]), and a good balance between the anterior and posterior areas of both feet was achieved. These results implied better stability and lower ulceration risk. The results of the dynamic analysis (Fig. 5 [5.8]) showed maintenance of the previous achievements and incorporation of the contact of the heel at the beginning of the step for the first time.

The patient was recommended to use a compression stocking daily. The pedobarographic results from September 2010 and
September 2012 showed the functional improvement obtained with this therapeutic measure (Fig. 6), which is clearly visible on the dynamic test, at which the toe footprints had appeared (Fig. 6 [6.2 versus 6.4 and 6.6 versus 6.8]). This improvement was also indicated by the increase in the foot contact time and the contact pressure value (both average and maximum). We also observed an increase in the contact area in the affected foot, progressive toe support, reduced oscillations, improved global equilibrium, a reduction in the stance phase time, and improved pressure distribution (average and maximum). We also observed a tendency for the shape of the footprint to be more similar to that of a healthy subject. Finally, we analyzed the progression over time of the static and dynamic values of the different variables obtained during the pedobarographic and gait measurements. 


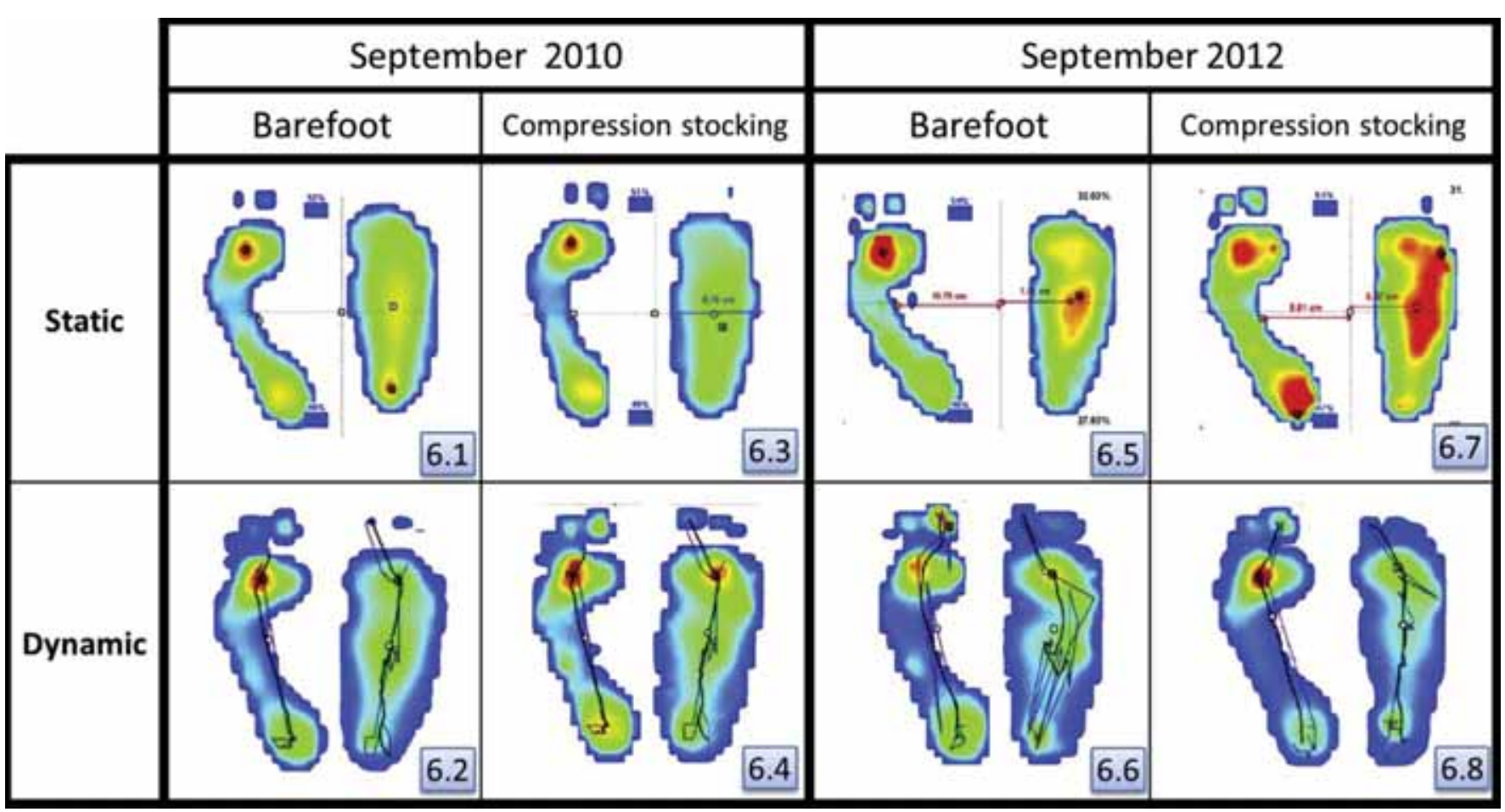

Fig. 6. Comparative evolution of static and dynamic pressure distributions with the patient barefoot and wearing a compression socking.

Static Analysis

The progression over time of the maximal pressure on both feet is presented in Fig. 7. In February 2010, the healthy foot bore approximately 4 times more pressure than the affected foot. This finding indicated that, in the static position, the patient was displacing her weight toward the nonaffected foot because she felt more comfortable and experienced less pain. However, this overload on the healthy foot could have had adverse effects, such as discomfort in the hips or knees. Nevertheless, as time progressed, the peak pressures of the affected foot and the healthy foot approached each other, indirectly indicating an improvement in balance, although the healthy foot always supported a slightly greater pressure.

The results showed that the affected foot was able to support greater average pressures with time (Fig. 8). In contrast, the pressure on the healthy foot decreased as the affected foot improved. The

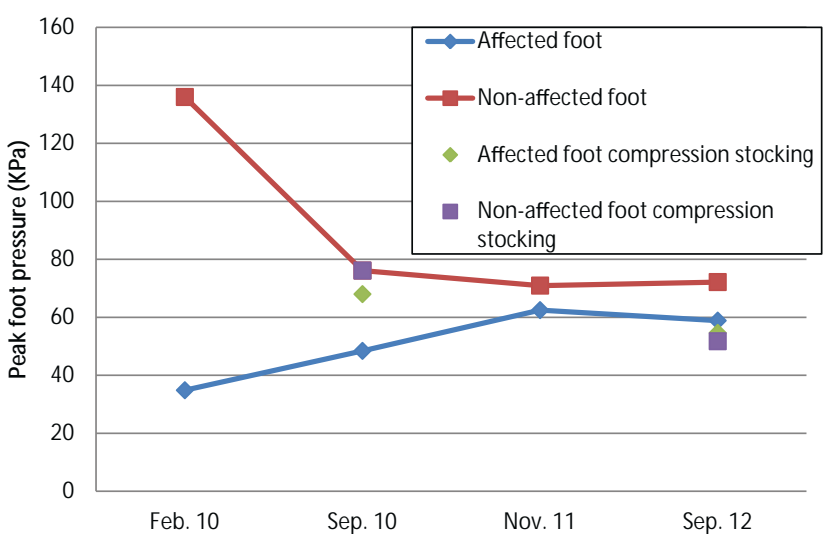

Fig. 7. Evolution of peak foot pressure during standing for the affected and nonaffected feet (static analysis). reconstructive surgical procedures conducted in August 2011 and August 2012 contributed strongly to this functional improvement in the pressure distribution.

The temporal progress of the contact surface (Fig. 9) did not show significant changes in the healthy foot but did show a noticeable increase in the affected foot. The second surgical procedure on the affected foot in August 2011 slightly reduced the plantar foot contact area and changed its morphology from oval- to paddle-shaped. The third surgery, in August 2012, helped stabilize the contact surface of the affected foot.

The loading time (Fig. 10) was initially altered because the healthy foot tried to compensate for the incorrect position of the affected foot, displacing the load to the heel. However, as time evolved, the loading time had equilibrated between both feet and between the forefoot and hindfoot.

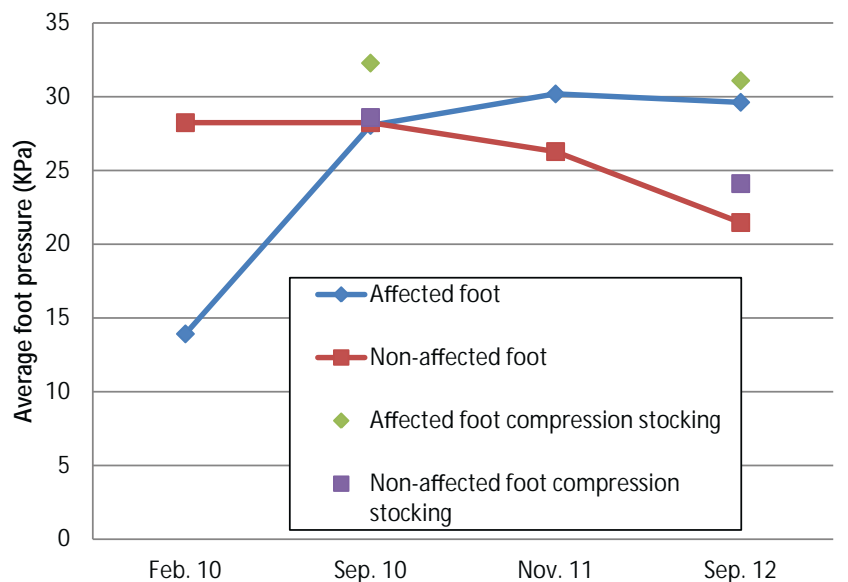

Fig. 8. Average foot pressure during standing in the affected and nonaffected feet (static analysis). 


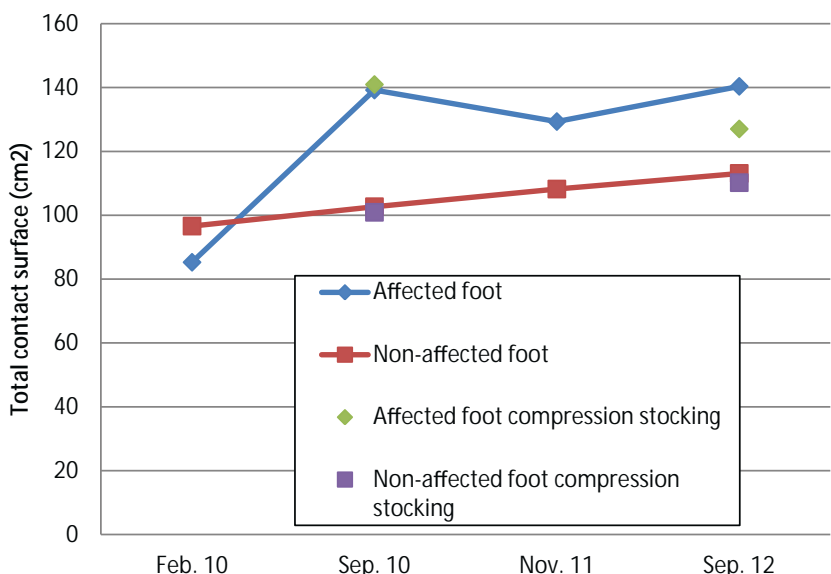

Fig. 9. Evolution of total contact surface $\left(\mathrm{cm}^{2}\right)$ in the affected and nonaffected feet during standing (static analysis).

\section{Dynamic Analysis}

In September 2010, the peak pressure (Fig. 11) was quite similar in both feet, although the shapes of the footprints were completely different. The video sequence (Supplemental Video S2) corresponding to this measurement indicated that the affected foot did not have a fast straight track but oscillated in a central critical area longer than the normal time. This implies an overload in that area, which could provoke the development of ulcerations over time. In the subsequent records, the peak pressure was more similar between the 2 feet, although it was always greater in the healthy foot. Similar findings were obtained in the static position.

The contact surface (Fig. 12) in the nonaffected foot showed virtually no changes over time. In the affected foot, the contact area increased progressively until an equilibrium was reached. The footprint contact surface and shape during gait was a key parameter of patient stability. The step time in both feet varied in parallel (Fig. 13). The results have clearly indicated that the functional improvement in the affected foot allowed the patient to walk faster by reducing the step time.

The use of pressure therapy to improve the surgical results is controversial. In our patient, we found that pressure stockings considerably improved the pedobarographic static and dynamic analysis results (Fig. 6). This was possible because the stocking held

\section{Loading Time (\%)}

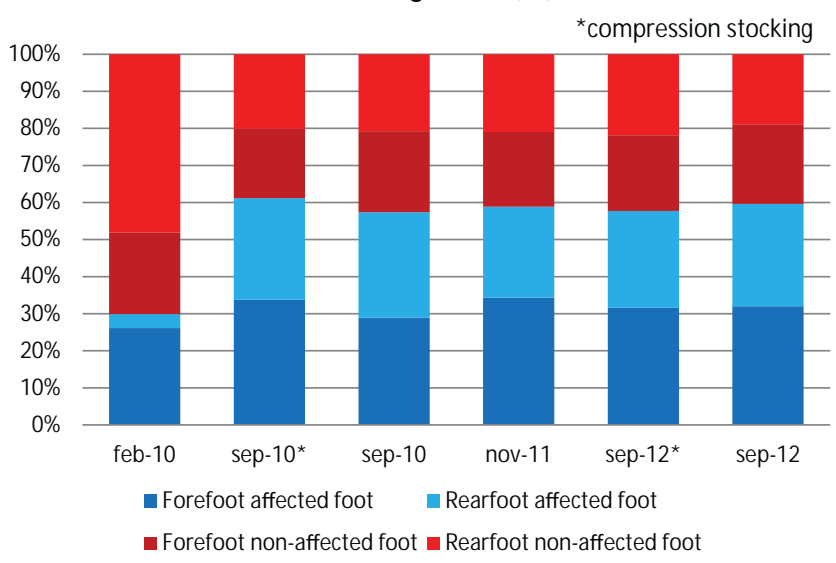

Fig. 10. Percentage of loading time during standing in the affected and nonaffected feet (static analysis).

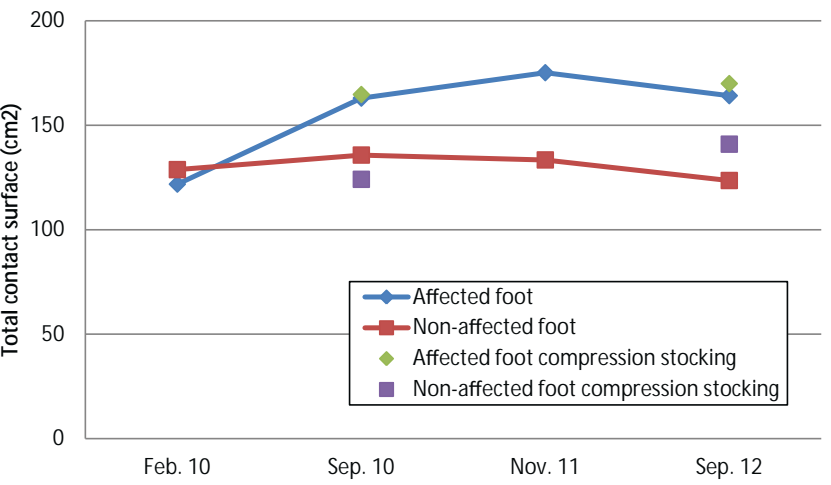

Fig. 11. Evolution of total contact surface $\left(\mathrm{cm}^{2}\right)$ in the affected and nonaffected feet (dynamic analysis).

the tissues that were subjected to weightbearing, which prevented their excessive movement. The pressure stocking also made the affected foot more similar to the nonaffected one.

\section{Gait Analysis}

The patient's gait was recorded in November 2011 and September 2012. On both occasions, the measurements were obtained with the patient both barefoot and wearing special insoles and shoes. No significant changes were found in the data comparison against the OptoGait $^{\circledR}$ database (Woodway World). Under normal conditions, the percentage of time of the stance and swing phases is approximately $60 \%$ for the former and $40 \%$ for the latter (11). The patient showed the same percentages in both cases.

\section{Discussion}

It is well known that the heel plays a vital role in normal ambulation. The heel is the first part of the foot to strike the ground during walking and acts as a fulcrum to disperse the vertical force toward the toes (9). Hidalgo and Shaw (15) classified foot injuries into 3 types: type I consists of limited soft tissue loss; type II has major soft tissue loss; and type III has bony damage, in addition to major soft tissue loss. Our patient's case corresponded to type III.

No significant differences have been found between reconstruction with reinnervated and noninnervated flaps. Both fasciocutaneous and skin-grafted muscle flaps, whether reinnervated or noninnervated, can be successfully used for weightbearing foot reconstruction. Neither of these flap types should be considered in the presence of peripheral neuropathy. The present patient had good

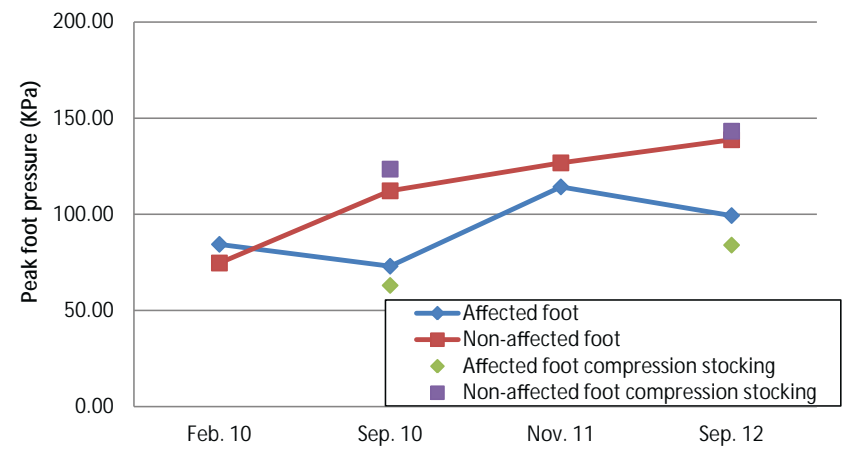

Fig. 12. Peak foot contact pressures $(\mathrm{kPa})$ in the affected and nonaffected feet (dynamic analysis). 


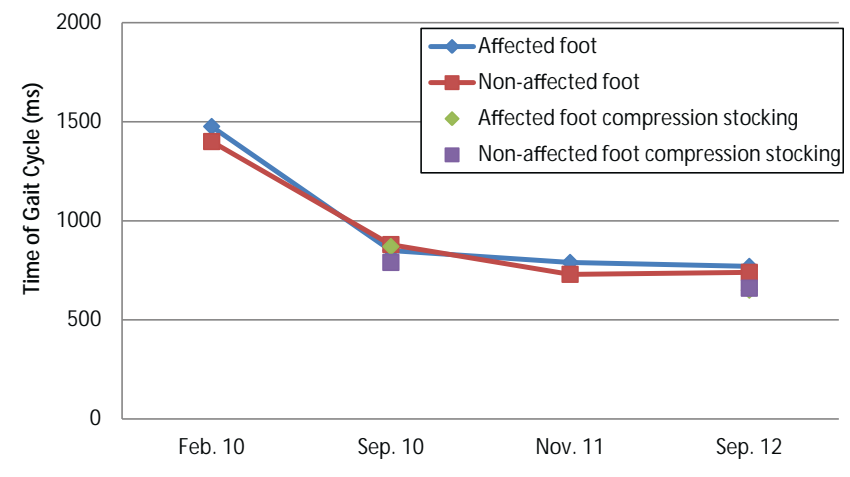

Fig. 13. Step time (ms) in the affected and nonaffected feet (dynamic analysis).

deep pressure sensation and thus was considered a good candidate for free-flap reconstruction (16-20).

The late functional results have been evaluated and reported in very few studies (21,22). May et al (23) used Harris mat footprints and footprint force-plate analysis to perform a detailed analysis of gait in trauma patients whose foot defects had been reconstructed with free muscle flaps and skin grafts. However, the addition of 3-dimensional motion analysis has provided additional highquality objective data. Goldberg et al (7) used oil foot-mat prints and gait analysis to evaluate the long-term results in trauma patients. In their study, gait was recorded using a video camera and was independently analyzed (7). Modern gait analysis units use more sophisticated camera systems and software programs that offer a more detailed analysis of gait disturbances. Dynamic pedobarography has mostly replaced the conventional footprint analysis with the Harris mat technique. Our report shows how kinetic and kinematic systems can help surgeons evaluate surgical functional reconstruction of the foot and complement clinical information (13).

In addition to the initial surgical coverage in November 2009, 2 interventions were conducted on the reconstructed foot in August 2011 and August 2012 to correct the shape of its footprint and decrease the global volume of the tissues. However, the contact surface between the plantar foot and the floor was drastically changed. This modification of the foot morphology caused an important change in the weightbearing pattern, which, in turn, resulted in a mechanical problem (Fig. 14).

The pedobarographic results have clearly shown that the affected foot's footprint initially had an oval shape, which progressively approached the shape of the nonaffected foot. Although the measured peak pressures were similar for both feet, their location in the affected foot changed over time from the central area to the physiologic metatarsal and heel areas. Fig. 5 clearly demonstrates that the clinical changes over time are small and difficult to

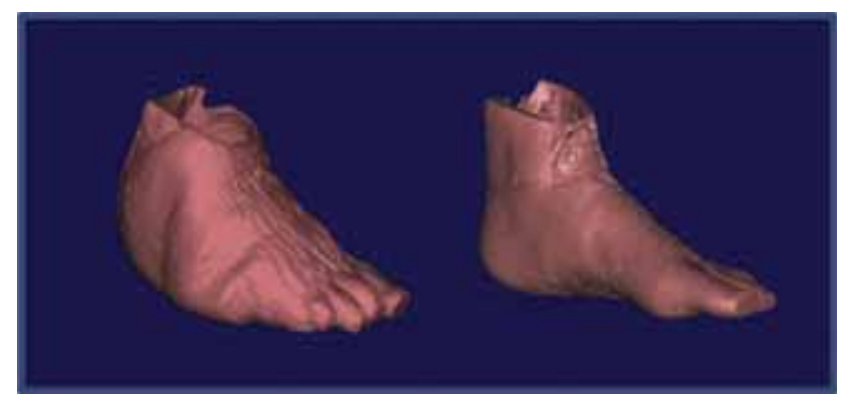

Fig. 14. View of reconstructed foot (Right) and nonaffected foot (Left). appreciate with the naked eye. In contrast, pedobarographic images provide additional and highly valuable information that can help determine whether the goals pursued before surgery have been reached. These images also provide an objective tool to evaluate whether the patient has improved over time, and they allow problems to be anticipated. More specifically, the pedobarographic images will corroborate the changes in the footprint and patient patterns of standing and walking before and after the surgeries. Pedobarographic measurements were not used to plan the surgical procedures, although they have served as a basis to determine whether the functional outcomes have been achieved and as objective measurements of excessive tissue.

Additionally, proper footwear protects reconstructed weightbearing surfaces by providing additional padding, reducing shear forces, or shifting the weight to a more stable area. The proper use of footwear can also prevent recurrent breakdown. Lord et al (12) suggested that the measurement of pressure beneath the foot could play a useful role in patient management by making it possible to provide custom-made pressure-relieving insoles. For our patient, insoles were designed using superficial 3-dimensional scanning of both feet and fast prototyping over a polyethylene block reinforced with carbon. The insoles were adapted at each follow-up visit to the morphologic changes in the patient's foot, which improved her outcome.

The medical team analyzed, not only the clinical results, but also the functional aspects of the patient's recovery before and after the successive surgical interventions. Thus, the postoperative functional results were observed in the subsequent pedobarographic and gait analysis tests. This method has also been used in other patients to compare the functional results after foot surgery and the results in patients with peripheral neuropathy with foot involvement.

In conclusion, in the present study, pedobarography was a useful tool for functional surgical evaluation, patient follow-up examinations, and pressure ulcer prevention. It also allowed for monitoring and comparison of the same patient or other patients. This method provides objective, quantifiable, additional and highly valuable information about the patient's functional clinical course and is superior to the simple clinical observations of the patient. Moreover, the quantitative data obtained with this technology can be used to evaluate the mechanical status of the internal foot tissues through finite element analysis (10). The pedobarographic results revealed the patient's functional and clinical improvements in both static and dynamic situations. The pedobarographic images corroborated the physiologic correction of the footprint and the patient's method of standing and walking after the surgeries. Furthermore, the use of pressure stockings and specific insoles improved all the parameters recorded in the patient and optimized the surgical functional results of the plantar foot surgeries. The patient's gait analysis results were comparable to those from a healthy population.

This is a new, objective, recordable, reproducible, and easy-to-use method that can help surgeons evaluate the functional results of plantar foot surgery. Orthoses should be used to correct the load distribution and pressure points underneath the reconstructed feet.

\section{Supplementary Material}

Supplementary material associated with this article can be found in the online version at www.jfas.org (http://dx.doi.org/10.1053/j.jfas. 2016.01.038).

\section{References}

1. Hanna R, Austin R. Lower-Extremity Injuries in Motorcycle Crashes In NHTSA Technical Report HS-810 982, U.S. Department of Transportation, National Highway Traffic Safety Administration, Washington DC, 2008. 
2. O'Brien BM, MacLeod AM, Hayhurst JW, Morrison WA. Successful transfer of a large island flap from the groin to the foot by microvascular anastomoses. Plast Reconstr Surg 52:271-278, 1973.

3. Jeng S, Wei F. Classification and reconstructive options in foot plantar skin avulsion injuries. Plast Reconstr Surg 99:1695-1703, 1997.

4. Hollenbeck ST, Woo S, Komatsu I, Erdmann D, Zenn MR, Levin LS. Longitudinal outcomes and application of the subunit principle to 165 foot and ankle free tissue transfers. Plast Reconstr Surg 125:924-934, 2010.

5. Aulivola B, Hile CN, Hamdan AD, Sheahan MG, Veraldi JR, Skillman JJ, Campbell DR, Scovell SD, LoGerfo FW, Pomposelli FB Jr. Major lower extremity amputation: outcome of a modern series. Arch Surg 139:395-399, 2004.

6. Meyer-Marcotty MV, Sutmoeller K, Kopp J, Vogt PM. Postoperative insolepaedobarographic gait analysis for patients with flap coverages of weightbearing and non-weight-bearing areas of the foot. J Plast Reconstr Aesthet Surg 65:482-488, 2012.

7. Goldberg JA, Adkins P, Tsai T. Microvascular reconstruction of the foot: weightbearing patterns, gait analysis, and long-term follow-up. Plast Reconstr Surg 92:904-911, 1993.

8. Villa Moreno A, Gutiérrez Gutierrérrez E, Pérez Moreno JC. Consideraciones para el análisis de la marcha humana. Técnicas de videogrametría, electromiografía y dinamometría. Rev Ingeniería 3:16-26, 2008.

9. Öztürk S, Bayram Y, Möhür H, Deveci M, Sengezer M. Evaluation of late functional results of patients treated with free muscle flaps for heel defects caused by landmine explosions. Plast Reconstr Surg 116:1926-1936, 2005.

10. Jordán-Palomar EI, Rey-Vasalo J. Diseño de estudio para la correlación de los datos de reconstrucción en 3D a partir de resonancia magnética y simulación computacional tras la reconstrucción plantar en zona de apoyo. Cir Plást Iberolatinoam 41:11-19, 2015.
11. Camp Faulí A, Montiel Parreño E, Poveda Verdú D, Salvador Palacios L, Orgilés Barcelo C, Faulí Marín A. Estudio dinámico de la presión en el pie: podobarografía. Av Diabetol 22:54-61, 2006.

12. Lord M, Reynolds DP, Hughes JR. Foot pressure measurement: a review of clinical findings. J Biomed Eng 8:283-294, 1986.

13. Uustal H, Baerga E. Prosthetics and orthotics: gait analysis. In: Physical Medicine and Rehabilitation Board Review, pp. 409-487, edited by S Cuccurullo, Demos Medical Publishing, New York, 2004.

14. O'Malley M, de Paor DL. Kinematic analysis of human walking gait. Med Eng Biol Comput 31:392-398, 1993.

15. Hidalgo DA, Shaw WW. Anatomic basis of plantar flap design. Plast Reconstr Surg 78:627-636, 1986.

16. Harrison DH, Morgan BD. The instep island flap to resurface plantar defects. Br J Plast Surg 34:315-318, 1981.

17. Haug M, Valderrabano V, Rieger U, Pierer G, Schaefer D. [Anatomically and biomechanically based treatment algorithm for foot and ankle soft tissue reconstruction]. Handchir Mikrochir Plast Chir 40:377-385, 2008.

18. Libermanis O. Replantation of the heel pad. Plast Reconstr Surg 92:537-539, 1993.

19. Sommerlad BC, McGrouther DA. Resurfacing the sole: long-term follow-up and comparison of techniques. Br J Plast Surg 31:107-116, 1978.

20. Gaulke R, Partecke BD. [Follow-up and gait study in patients with plastic-surgerytreated foot sole defects]. Unfallchirug 97:47-53, 1994.

21. Potparić Z, Rajacić N. Long-term results of weight-bearing foot reconstruction with non-innervated and reinnervated free flaps. Br J Plast Surg 50:176-181, 1997.

22. Milanov NO, Adamyan RT. Functional results of microsurgical reconstruction of plantar defects. Ann Plast Surg 32:52-56, 1994.

23. May JW, Halls MJ, Simon SR. Free microvascular muscle flaps with skin graft reconstruction of extensive defects of the foot: a clinical and gait analysis study. Plast Reconstr Surg 75:627-641, 1985. 